\title{
Impedance spectroscopy on lithium ion cells for automotive applications with optimized measurement duration and frequency resolution

\author{
Peter Haußmann ${ }^{1}$, Joachim Melbert ${ }^{1}$
}

${ }^{1}$ Forschungsgruppe Kfz-Elektronik, Ruhr-Universität Bochum, Universitätsstr. 150, 44801 Bochum

peter.haussmann@est.rub.de

\begin{abstract}
A new impedance spectroscopy measurement procedure for automotive battery cells is presented, which is based on waveform shaping. The method is optimized towards short measurement duration, high excitation energy and increased frequency resolution and overcomes the limitations of established methods. For a given spectral amplitude profile, the corresponding time domain waveform is derived from the inverse discrete Fourier transform. Applying an identical initial phase angle for each frequency component, the resulting signal exhibits a high peak-to-peak amplitude at relatively low total excitation energy. This limits the maximum allowed energy for linear excitation. Altering the phase angles randomly spreads the excitation energy across the complete measurement duration. Thereby, linearity is preserved at higher excitation energy. By including window functions in the synthesis concept, spectral leakage is reduced without changing the spectral signal amplitude in the frequency range of interest. A time domain waveform optimized for impedance spectroscopy on lithium ion cells is synthesized based on the proposed approach and evaluated on real automotive cells. The resulting impedance data show good concordance with established standard measurement procedures at significantly reduced measurement duration and charge throughput. Additionally, increased frequency resolution is achieved, enhancing the level of detail of the obtained impedance data. The method is used for improved localization of aging effects in the cells, without further stress of the cells by the measurement procedure.
\end{abstract}

Keywords: electrochemical impedance spectroscopy, broadband excitation, waveform shaping, signal synthesis, windowed excitation

\section{Introduction}

Lithium ion cells for the use in electric vehicles are subject to ongoing research, aiming to reach higher energy densities and longer lifetimes while maintaining safe operation. To reach these goals, cell chemistry is steadily optimized. To evaluate the performance of a cell technology, benchmark and cycle life studies are conducted. These studies rely on precise and reproducable characterization methods to quantify the cell's key properties, like its internal resistance and capacitance, which are strongly influenced by the material composition of the cell. Therefore, analyzing the aging behaviour of various materials seperately and its localization in the cell is mandatory.

Electrochemical impedance spectrosopy (EIS) is an established method in the characterization of electrochemical systems. By analyzing measurement data of current and voltage of a lithium ion cell, the frequency dependent complex impedance $\underline{Z}(j \omega)$ can be calculated.
The application of EIS on lithium ion cells for automotive energy storage is a challenging task. Compared to other electrochemical systems, the internal impedance of standard automotive lithium ion cell is usually in the $m \Omega$ range, requiring high current amplitudes.

The most established approach is based on sinusoidal excitation with a given amplitude, which ensures quasi linear response. The impedance is measured at a discrete number of frequencies, one at a time. This method is robust against influences of noise, as the spectral excitation power is concentrated on a single frequency. However, especially for low frequencies in the $\mathrm{mHz}$ range, long measurement durations are required.

In impedance measurements, a dense frequency resolution is desirable. For the single frequency approach, a large number of frequencies has to be chosen. Since several periods at each frequency need to be analyzed sequentially, the complete measurement procedure is very time-consuming, especially at low frequencies. At measurement duration of several hours, time-invariance of the DUT cannot be guaranteed [1], [2]. 
An alternative apporach optimized towards short measurement duration is based on pulse excitation. Opposed to the stepped-sine approach, multiple frequencies are covered simultaneously. The frequency domain characteristic results from the time domain pulse shape. Although rectangularly shaped pulses can be synthesized on almost any test equipment, their spectra are less useful due to the non uniform power density. Using sophisticated test equipment capable of arbitrary waveform generation, spectrally optimized pulse shapes like sinc $(\sin (\mathrm{x}) / \mathrm{x})$ pulses are favourable [2]. By employing synthetic excitation signals based on defined spectral amplitude profiles, further optimization of the waveform is possible [3]. However, excitation energy is concentrated around the main pulse slopes. Considering the long measurement duration required for the analysis of low frequency EIS, signal energy is still limited by the boundary condition for linear excitation.

Broadband excitation based on band limited noise signals avoids temporal energy concentration. However, spectral amplitude is randomly distributed across the observed frequency range. Therefore, suitable excitation is not guaranteed for all frequencies of interest [4].

In this work, a novel excitation signal generation method is presented, aiming to combine the advantageous spectral amplitude properties of pulse signals with wide-spread time domain characteristics typical of noise signals. This is achieved using a waveform shaping approach based on the inverse discrete foruier transform (IDFT) in the digital signal synthesis [5], supplemented by the inherent use of window functions.

\section{Fundamentals}

The impedance of any electrochemical system can be defined as the voltage response following a current excitation:

$$
\underline{Z}(j \omega)=\frac{v(j \omega)}{\underline{i(j \omega)}}=|\underline{Z}| e^{j \Delta \varphi}
$$

Impedance measurements on a device-undertest (DUT) are performed by applying an excitation signal and measuring the system response. In theory, current and voltage excitation can be applied equivalently. In the case of low internal impedance in the $\mathrm{m} \Omega$ range and capacitive behavior, voltage excitation is not feasible. This is the case for contemporary automotive lithium ion cells. For that reason, current controlled excitation is preferred.
For the interpretation of EIS results, the measured impedance is fitted to an electrochemically motivated equivalent circuit. This allows a link between the measured electrical characteristics and the underlying electrochemical processes inside the cell. The effects observable in automotive lithium ion cells can roughly be separated according to their characteristic frequency range. In the $\mathrm{kHz}$ region, inductive behavior resulting from the cell's geometry can typically be identified. The $\mathrm{Hz}$ range is dominated by double layer capacitance effects at the solid electrolyte interphase (SEI) formed between electrodes and electrolyte. In comparison, lithium ion diffusion processes through the electrolyte are slower by another order of magnitude, determining the impedance in the $\mathrm{mHz}$ range [6].

In Fig. 1, a typical impedance spectrum of a lithium ion cell is depicted in the Nyquist representation, describing the relation of the real and imaginary part of the impedance.

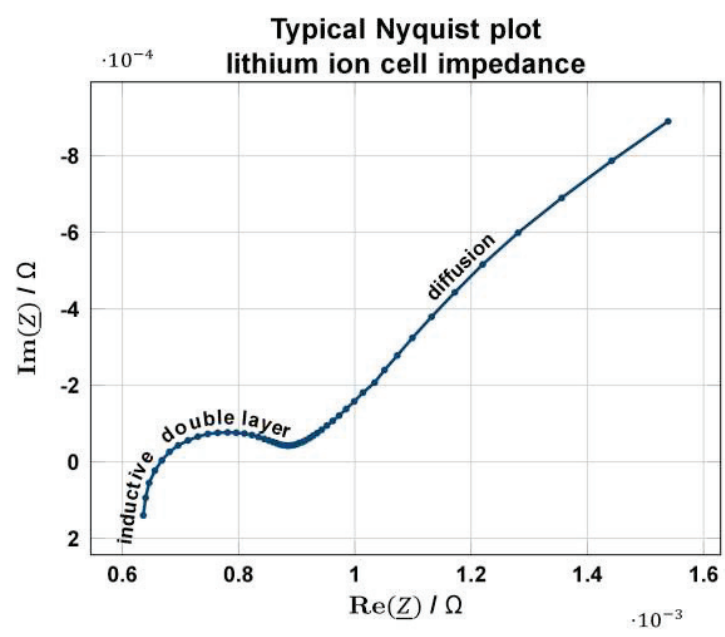

Fig. 1 Nyquist plot of lithium ion cell impedance from $10 \mathrm{mHz}$ (top right) to $1 \mathrm{kHz}$ (bottom left). Note the inverse imaginary axis chosen for the reader's convenience.

For robust model fitting, good impedance data quality and integrity is required. Data quality refers to frequency resolution and the signalto-noise ratio, which is limited by the excitation signal amplitude.

Furthermore, the model fitting approach implicitly requires that the underlying system has to be linear, time-invariant and stationary. These conditions are not necessarily satisfied by a lithium ion cell. Typical violations are nonlinear behavior due to exceeding an adequate value of the excitation amplitude, or time variance due to temperature or state-of-charge changes during the measurement. If one or more of these effects occur, the measurement 
data are unsuitable for equivalent circuit fitting. Therefore, verification of the EIS results integrity is required.

The Kramers-Kronig relations describe the interdependency of the real and imaginary part of causal LTI-system (linear time invariant) transfer functions in system theory. A KramersKronig compliance test method applicable to EIS results is presented in [7]. The algorithm is employed to validate the measurement results in this work.

As the impedance is defined in the frequency domain, spectra calculation usually involves a time-frequency domain transform like the discrete Fourier transform (DFT). For an input time domain signal consisting of $k$ samples at the sampling frequency $f_{S}$, the minimum frequency $f_{\min }$ considered by the DFT equals the reciprocal value of the total sampling duration.

$$
f_{\min }=\frac{f_{S}}{k}=\frac{1}{T_{\text {meas }}}
$$

According to the well-known Nyquist theorem, the upper frequency bound is calculated as:

$$
f_{\max }=\frac{f_{S}}{2}
$$

Therefore, in order to investigate the DUT's impedance at frequency $f_{i}$, the following constraints have to be met:

$$
\begin{gathered}
f_{S} \geq 2 f_{i} \\
T_{\text {meas }} \geq \frac{1}{f_{i}}
\end{gathered}
$$

The required values for the sampling frequency derived from Eq. (4) are not an issue in state of the art measurement systems, considering the typical frequency range of interest. However, in the $\mathrm{mHz}$ range, the constraint in Eq. (5) regarding $T_{\text {meas }}$ might lead to extremely long measurement durations.

In theory, $f_{\min }=T_{\text {meas }}^{-1}$ is the minimum frequency analyzed by the DFT. However, signal components exhibiting a frequency of lower than $f_{\text {min }}$ contribute to the low frequency range as undesired artifacts of the DFT spectrum due to spectral leakage. To avoid measurement errors in practical scenarios, the number of periods $n$ analyzed per frequency is usually chosen as a multiple of the maximum period length of interest, resulting in even longer measurement durations. In typical measurement scenarios, $n \geq 3$ is an adequate choice.

\section{Standard impedance spectroscopy measurement procedures}

A fundamental requirement for any EIS method is an adequate excitation of the DUT in the frequency range of interest. In most conventional approach, the DUT is stimulated at only one frequency at a time using sinusoidal excitation signals. In literature, this procedure is referred to as stepped-sine EIS. The entire excitation energy is concentrated on a single frequency, resulting in a convenient signal to noise ratio.

The complete measurement duration can be calculated using the following equation, assuming $\mathrm{n}$ periods of $\mathrm{m}$ different frequencies:

$$
T_{\text {Meas,total,sin }}=\sum_{i=1}^{m} \frac{n}{f_{i}}
$$

In the $\mathrm{mHz}$-range, signal periods last up to 15 minutes. Depending on the desired frequency range and resolution, typical values of $T_{\text {Meas,total }}$ range from several hours up to days. In cycle life studies, such long time frames are not available for characterization. Furthermore, time invariance cannot be assumed during long measurement durations.

In order to reduce measurement duration while preserving the same frequency resolution, broadband excitation using pulse waveforms can be applied. Opposed to sinusoidal signals, multiple frequency components are present in the excitation signal [1].

The spectral density characteristic determine the performance of a pulse waveform for EIS. For most commercial test equipment, only rectangular pulses are available, suitable for measurements with low demand on duration and charge throughput. Such excitation waveform provides a non-uniform power density across the frequency range of interest resulting which results into a poor signal to noise ratio.

Alternative pulse forms like sinc pulses enable a homogenous broadband excitation [2]. The signal synthesis can be performed in time domain based on the equation:

$$
x_{\text {sinc }}(t)=\frac{\sin (2 \pi f t)}{2 \pi f t}
$$

Equivalently, the time domain waveform of the sinc pulse can be interpreted as the result of the inverse Fourier transform of a rectangular spectral amplitude profile [8].

For pulse signals covering the complete frequency range of interest, the required measurement duration is calculated as a multiple of the period of the minimum frequency of interest: 


$$
T_{\text {Meas,total,pulse }}=\frac{n}{f_{\text {min }}}
$$

However, the spectral excitation energy is shared by all frequency components of the stimulus signal, offering a limited signal-tonoise ratio with respect to each frequency component. This can be overcome by using multiple excitation pulses covering different frequency ranges. If every pulse covers a relative bandwidth of one frequency decade, the overall measurement duration for a desired bandwidth of $l$ decades results in:

$$
T_{\text {Meas,total,pulse }}=\sum_{k=1}^{l} \frac{n}{10^{k-1} \cdot f_{\min }} \approx 1.1 \cdot \frac{n}{f_{\min }}
$$

A comparison between the equations (6) and (9) shows a significant reduction of measurement duration for the pulse EIS approach compared to stepped-sine procedures.

Even for spectrally optimized pulse shapes like sinc pulses, established time domain waveforms exhibit a low average signal energy at a high transient peak-to-peak amplitude, limiting the achievable quality of the measured impedance spectra due to linearity constraints.

\section{Time domain waveforms synthesis for arbitrary spectral amplitude profiles}

In this paper, a waveform shaping method is developed. The design flow can be used to synthesize time domain waveforms based on an arbitrary spectral amplitude profile $\underline{X}(f)$.

The time domain waveform $x(t)$ is synthesized by applying the IDFT to the spectral amplitude profile $\underline{X}(f)$.

The frequency sampling points $f_{i}$ of the profile are dependent on the total signal duration $T_{\text {sig }}$ and the sampling frequency $f_{s}$, in accordance with the aforementioned properties of the (I)DFT. For each frequency component $f_{i}$, the target amplitude $A_{i}$ is defined.

$$
A_{i}= \begin{cases}1, & \text { if } f_{\min } \leq f_{i} \leq f_{\max } \\ 0, & \text { otherwise }\end{cases}
$$

In the following, the target frequency domain excitation profile defined in Eq. (10) is used. It exhibits a rectangular shape with homogenous coverage from $f_{\min }$ up to $f_{\max }$. As noted in equations (11) and (12), a relative bandwidth of one frequency decade is covered, including a $10 \%$ margin above $f_{\max }$.

$$
\begin{gathered}
f_{\text {min }}=\frac{f_{s}}{100} \\
f_{\text {max }}=1.1 \frac{f_{s}}{10}
\end{gathered}
$$

The measurement duration is chosen to $T_{\text {sig }}=\frac{4}{f_{\text {min }}}$ in order to avoid errors due to low frequency artifacts. The sampling frequency $f_{s}$ may be chosen with respect to the frequency range of interest.

For the unambiguous definition of frequency domain signals, both amplitude information $A_{i}$ and the initial phase angle $\varphi_{i}$ are required. Initially, the phase angle is chosen to:

$$
\varphi_{i}=0
$$

The resulting waveform depicted in Fig. 2 resembles a frequency modulated sinc pulse and shows high temporal concentration of the excitation energy at the pulse's highest slopes, as expected in analogy to common pulse synthesis approaches.
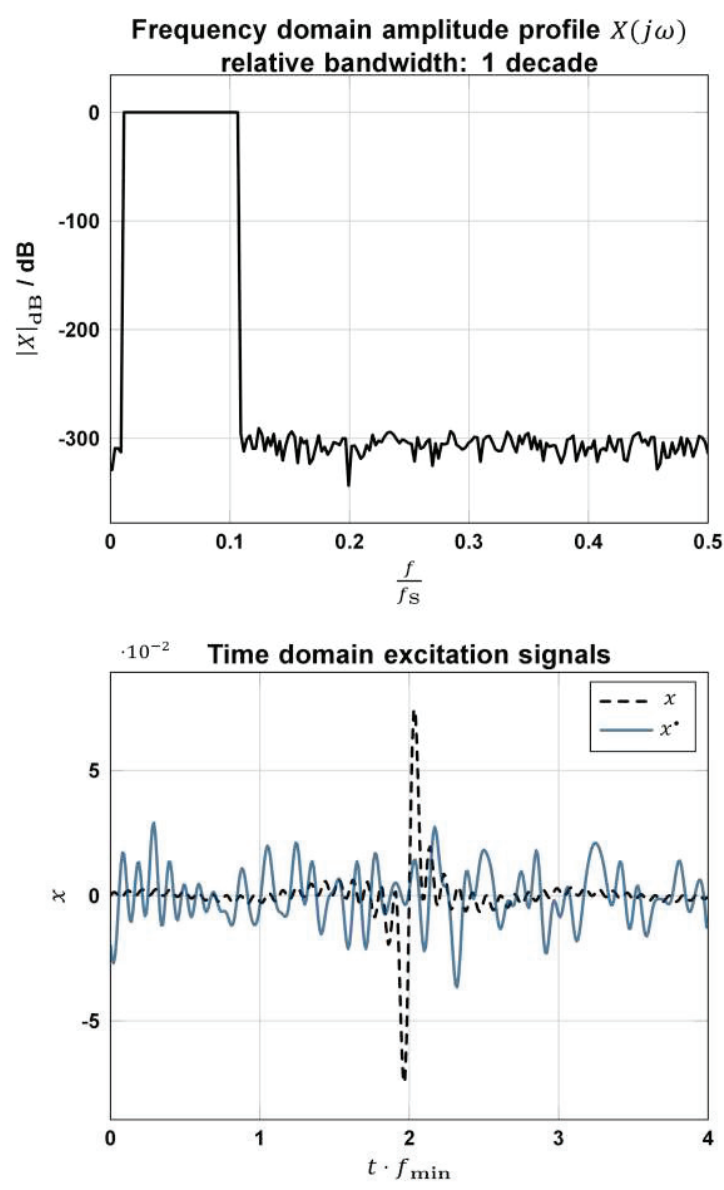

Fig. 2 top: normalized frequency domain amplitude profile for a relative bandwidth of one frequency decade. 
Bottom: time domain signals with identical spectral amplitude characteristics without (black) and with (blue) phase modification.

In EIS measurements, only the relative phase angle between voltage and current is relevant. Therefore, the absolute initial phase angle of each excitation frequency component may be altered arbitrarily. In this example, an individual random phase angle is chosen for every frequency component:

$$
\varphi_{i}=\operatorname{RANDOM}(0: 2 \pi)
$$

This leads to a broadened distribution of the signal energy in the time domain waveform. The transient peak-to-peak amplitude is significantly reduced at identical frequency domain characteristics. The corresponding waveform $x^{\bullet}(t)$ is depicted in Fig. 2.

\section{Spectral leakage reduction using pre- excitation window functions}

Due to the random phase shift of the frequency components, the first and last samples are generally not equal. Therefore, the time domain signal $x^{*}(t)$ is not periodically repeatable. This results in amplitude and phase errors due to spectral leakage in the DFT. In order to reduce the impact of this effect, $x^{\bullet}(t)$ can be multiplied by a window function, resulting in the post-synthesis windowed (PSW) signal $x^{\bullet}(t)$ :

$$
\begin{gathered}
x_{\mathrm{PSW}}^{\bullet}(t)=x^{\bullet}(t) \cdot h_{\text {win }}(t) \\
\vdots \\
\underline{X}_{\mathrm{PSW}}^{\bullet}(f)=\underline{X}^{\bullet}(f) * H_{\text {win }}(f)
\end{gathered}
$$

In this work, a narrow band Gaussian window function is used. The low window bandwidth is represented by a relatively broad time domain shape, as desired to maintain a widespread temporal energy distribution [9].

Besides the desired spectral leakage reduction, the use of a window function also influences the spectral amplitude $\left|\underline{X}_{\mathrm{PSW}}^{\bullet}(f)\right|$. The signal energy exhibits different temporal localization for every frequency, depending on the phase angle $\varphi_{i}$. Therefore, the waveform $x_{\mathrm{PSW}}^{\bullet}(t)$ does not exhibit the desired characteristics, as the window function reduces the spectral amplitude of each frequency to a different extent.

The frequency dependent attenuation can be avoided by integrating the window function into the signal synthesis flow. Intra-synthesis windowing (ISW) combines the spectral leakage reduction introduced by the window function with the frequency domain amplitude characteristics of the target profile. This is achieved by scaling the frequency domain signal $\underline{X}^{\bullet}(f)$ by a frequency dependent factor $\alpha(f)$ before applying the IDFT and the window function:

$$
\underline{X}_{\mathrm{ISW}}^{\bullet}(f)=\left(\underline{X}^{\bullet}(f) \cdot \alpha(f)\right) * H_{\text {win }}(f)
$$

The required scaling factor $\alpha(f)$ is determined using an iterative numeric optimization. The resulting waveform is referred to as $x_{\mathrm{ISW}}^{\bullet}(t)$.
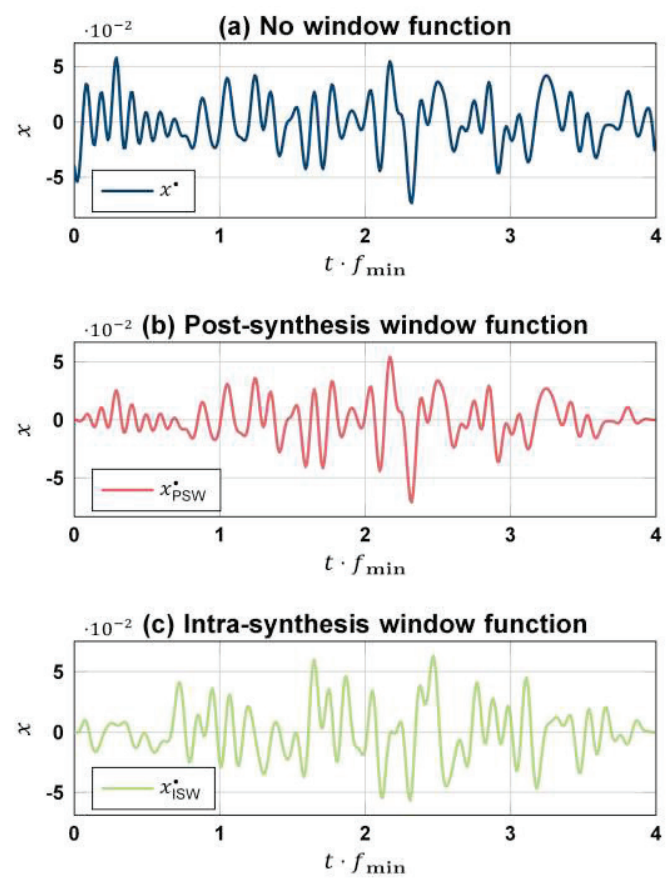

(d) Frequency domain comparison

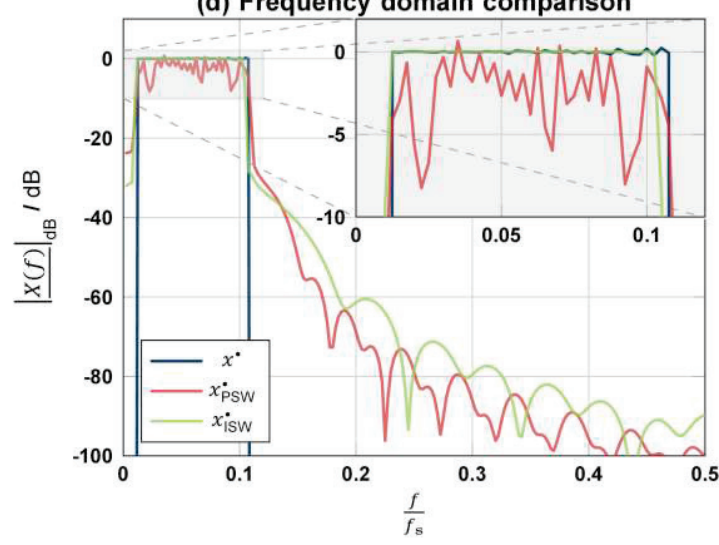

Fig. 3 Time domain waveforms with (a) no window function, post-synthesis window function (b) and intrasynthesis window function (c). In (d), the spectral amplitude of all waveforms is compared. 
In Fig. 3, the phase modified time domain signals $x^{\bullet}(t)$ (no windowing), $x_{\mathrm{PSW}}^{\bullet}(t)$ (post synthesis windowing) and $x_{\mathrm{ISW}}^{\cdot}(t)$ (intra synthesis windowing) are compared in time and frequency domain. All signals are normalized to a spectral amplitude of $0 \mathrm{~dB}$ across the desired frequency range.

While the time domain signal shape of $x_{\mathrm{PSW}}^{\bullet}(t)$ and are similar at first sight, the frequency-dependent attenuation of $\left|X_{\mathrm{PSW}}^{\bullet}(f)\right|$ is confirmed in the frequency domain comparison. The maximum amplitude loss is greater than $6 \mathrm{~dB}$, which reduces the achievable signal-to-noise ratio in measurement scenarios. The extended amplitude rescaling during the synthesis of $x_{\text {ISW }}^{*}(t)$ avoids any amplitude loss caused by the window function.

In Tab. 1, a short comparison of the time domain signals discussed in this paper is given. All signals are based on a one decade rectangular spectral amplitude profile. The most suitable waveform for EIS measurements is $x_{\text {ISW }}^{\bullet}(t)$, which includes peak amplitude reduction using phase modification and reduced spectral leakage due to the inherently applied window function. Compared to the unmodified signal $x(t)$, the transient peak-topeak amplitude of $x_{\text {ISW }}^{\cdot}(t)$ is reduced by $60 \%$ without altering the signal energy in the frequency range of interest. In EIS measurements, the spectral amplitude of the can be increased to the same extend without violating linearity constraints.

Therefore, $x_{\mathrm{ISW}}^{\cdot}(t)$ is used as excitation signal for the time domain EIS measurements presented in this work.

Tab. 1 Comparison between the discussed time domain waveforms with equal target spectral amplitude profile. + (positive), o (neutral), (-) negative

\begin{tabular}{|c|c|c|c|c|}
\hline Signal & $x$ & $x^{\bullet}$ & $x_{\mathrm{PSW}}^{\bullet}$ & $x_{\text {ISW }}^{\cdot}$ \\
\hline Rel. bandwidth & \multicolumn{5}{|c|}{1 decade } \\
\hline Phase shift & & $\mathrm{X}$ & $\mathrm{X}$ & $\mathrm{X}$ \\
\hline $\begin{array}{c}\text { Amplitude } \\
\text { flatness }\end{array}$ & + & + & - & + \\
\hline Spectral leakage & 0 & - & + & + \\
\hline $\begin{array}{c}\text { Peak-to-peak } \\
\text { amplitude relative } \\
\text { to } x\end{array}$ & 1 & 0.44 & 0.42 & 0.40 \\
\hline
\end{tabular}

\section{Measurement results}

For the practical evaluation of the proposed measurement procedure, a precision lithium ion single cell test unit developed by our research group was used [2]. It is equipped with a $600 \mathrm{~A}$ wideband linear discharge current sink along with a measurement control unit, enabling accurate measurements with errors below $0.1 \%$. Furthermore, arbitrary current profiles are supported at sampling rates up to $10 \mathrm{kHz}$.

The DUT is a single 26 Ah automotive cell for the use in plug-in hybrid vehicles (PHEV). All measurements are conducted at $25^{\circ} \mathrm{C}$ ambient temperature and $50 \%$ state of charge. In order to verify the spectral characteristics of the proposed waveform $x_{\text {ISW }}^{\cdot}(t)$, it is applied to the DUT as a current profile $i_{\text {DUT }}(t)$.

Both the target waveform and the measured signal are shown in time and frequency domain in Fig. 4. The synthesis concept is verified by the close consistency of the target and measured signal both in time and frequency domain.
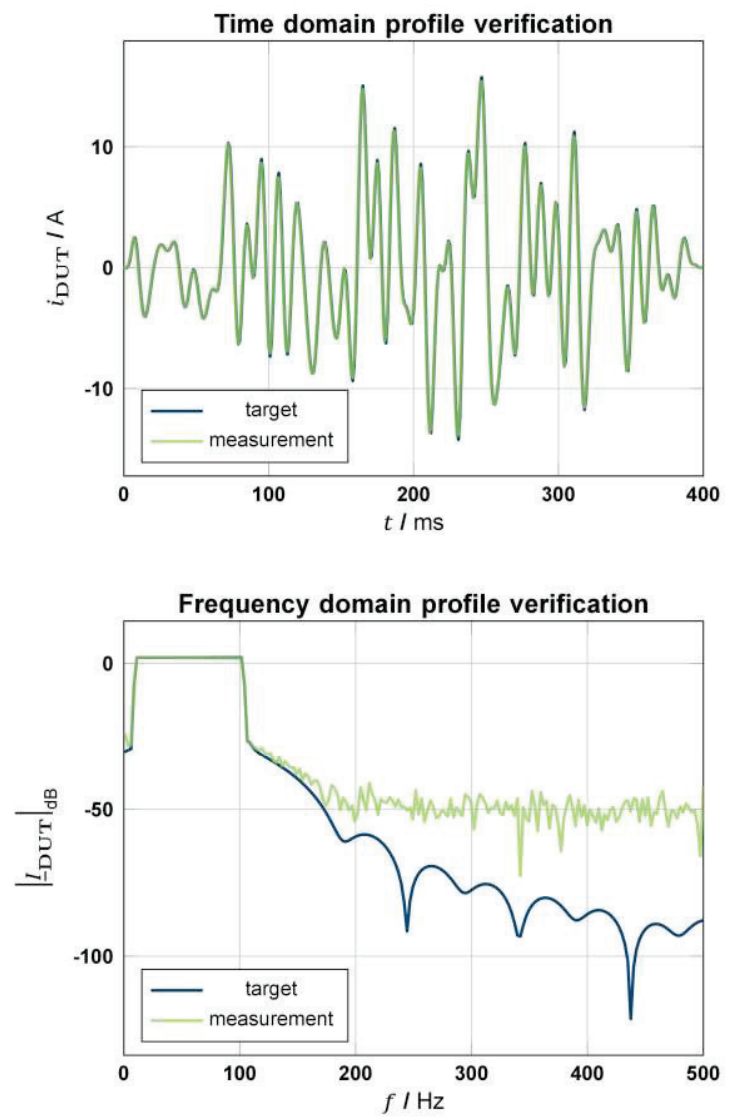

Fig. 4 Profile verification in time (top) and frequency domain (bottom). The measured signal (green) closely resembles the target profile across the entire target bandwidth $(10 \mathrm{~Hz}$ to $100 \mathrm{~Hz}$ ). 
To evaluate the suitability of the proposed waveform for EIS measurements, the DUT's impedance is measured in the frequency range from $10 \mathrm{mHz}$ to $1 \mathrm{kHz}$ using both the established stepped-sine approach (51 frequency points in 30 minutes) and the proposed measurement procedure based on $x_{\text {ISW }}^{\cdot}(t)$ in 5 different time scales at a total duration of 8 minutes. The Nyquist plots of the resulting impedance spectra are depicted in Fig. 5. The results of both procedures show good concordance, indicating the suitability of the proposed time domain method.

Due to the waveform's broadband excitation characteristics, frequency resolution may be chosen arbitrarily within the aforementioned limitations of the DFT.

Especially at low frequencies, the spectrum obtained from the optimized waveform is enriched by the increased frequency resolution.

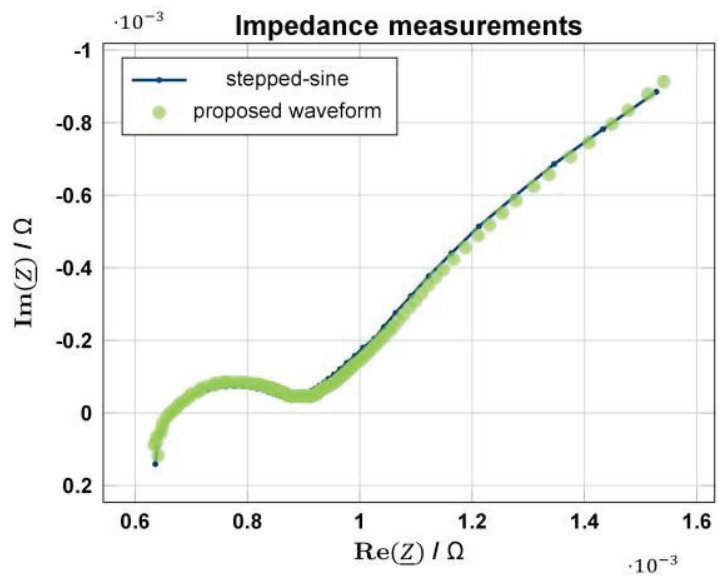

Fig. 5 Nyquist plots of the DUT's impedance measured using stepped sine (blue) and optimized waveform (green) approaches.

To evaluate the data quality of the measured spectra, a Kramers-Kronig compliance test is performed on the impedance data obtained using both methods. In the test procedure, an a priori Kramers-Kronig compliant impedance spectrum is calculated based on the real part of the measured spectrum. A deviation between the measured and the calculated imaginary part indicates a violation of the Kramers-Kronig constraints.

The imaginary part residual distribution for both impedance spectra is depicted in Fig. 6. For both spectra, a homogeneous distribution of the residual can be seen. As no significant deviation can be recognized, the KramersKronig compliance of both spectra is confirmed. The observed residual is caused by measurement noise. Due to the increased available excitation energy in stepped-sine measurements, a lower residual is achieved compared to measurements based on the proposed waveform.

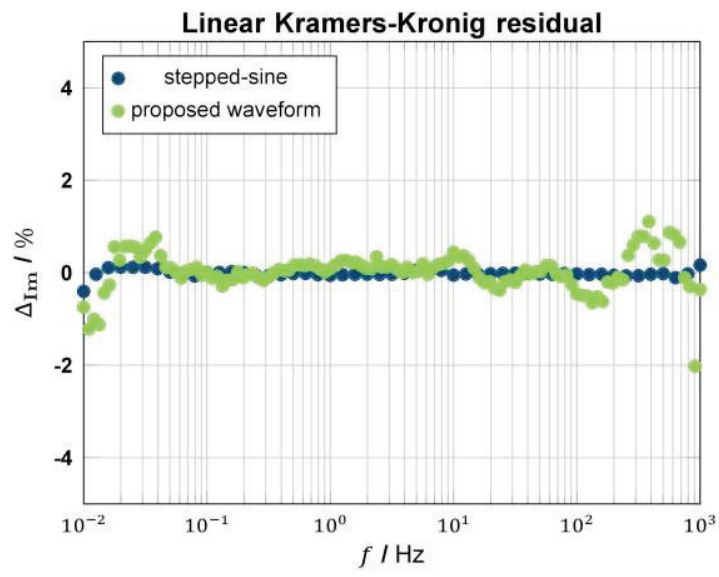

Fig. 6 Linear Kramers-Kronig residual for impedance spectra measured using stepped-sine (blue) and waveform shaping (green) methods.

In Tab. 2, a quantitative comparison of both measurement procedures is given. At significantly reduced measurement duration and charge throughput, excitation based on the proposed waveform shaping approach yields EIS results equivalent to spectra obtained using the standard stepped-sine EIS procedure. The slightly increased residual for the proposed method does not compromise the result's integrity, especially when taking the aforementioned improvements into account.

Tab. 2 Quantitative comparison of steppedsine and optimized time domain EIS measurements on an automotive lithium ion cell

\begin{tabular}{|c|c|c|}
\hline Measurement & Stepped Sine & \multicolumn{1}{|c|}{$x_{\mathrm{ISW}}^{\bullet}(t)$} \\
\hline $\begin{array}{c}\text { Frequency } \\
\text { range }\end{array}$ & \multicolumn{2}{|c|}{$10 \mathrm{mHz}-1 \mathrm{kHz}$} \\
\hline $\begin{array}{c}\text { Frequency } \\
\text { resolution }\end{array}$ & $\begin{array}{c}10 \text { points per } \\
\text { decade }\end{array}$ & $\begin{array}{c}24 \text { points per } \\
\text { decade } \\
(+140 \%)\end{array}$ \\
\hline Total duration & 30 minutes & $\begin{array}{c}8 \text { minutes } \\
(-73 \%)\end{array}$ \\
\hline $\begin{array}{c}\text { Charge } \\
\text { throughput }\end{array}$ & $2,41 \mathrm{Ah}$ & $\begin{array}{c}0,27 \mathrm{Ah} \\
(-88 \%)\end{array}$ \\
\hline $\begin{array}{c}\text { Mean KK } \\
\text { residual }\end{array}$ & $0,05 \%$ & $0,28 \%$ \\
\hline
\end{tabular}

${ }^{*}$ Frequency resolution is chosen arbitrarily 


\section{Conclusion}

A waveform shaping method to generate broadband time domain signals was developed and optimized for high signal power at low peak-to-peak amplitudes. Compared to common pulse waveforms, a $60 \%$ decrease in peak-to-peak amplitude is achieved at a relative bandwidth of 1 decade. The overall signal amplitude can be increased to the same extent, still enabling linear excitation of the DUT. The concept was verified in simulation and measurement.

EIS results based on the optimized time domain waveform show good concordance with reference stepped sine EIS measurements, reducing measurement duration by $73 \%$ and charge throughput by $88 \%$.

In comparison to equivalent pulse excitation schemes, a higher excitation amplitude is achieved by deconcentrating signal energy in the time domain. Spectral distortion caused by spectral leakage can be avoided by applying window functions within the waveform shaping flow.

To some extent, the proposed pulse shapes resemble typical current profiles measured in real automotive driving scenarios. Despite the limited spectral amplitude control in real measurement scenarios, passive on-board EIS implementations are possible, improving the state-of-charge and state-of-health estimation algorithms in the next generation of battery management systems [10].

\section{References}

[1] D. Klotz, M. Schönleber, J. P. Schmidt, and E. Ivers-Tiffée, "New approach for the calculation of impedance spectra out of time domain data," Electrochimica Acta, vol. 56, no. 24, pp. 8763-8769, 2011; doi:

10.1016/j.electacta.2011.07.096

[2] N. Lohmann, P. Weßkamp, P. Haußmann, J. Melbert, and T. Musch, "Electrochemical impedance spectroscopy for lithium-ion cells: Test equipment and procedures for aging and fast characterization in time and frequency domain," Journal of Power Sources, vol. 273, pp. 613-623, 2015; doi: 10.1016/j.jpowsour.2014.09.132

[3] T. Guerrero Cervera, A. P. Vega-Leal, G. Adame García, and J. Brey Sánchez, "PEMFC impedance spectroscopy using synthetic wide-band signals," International Journal of Hydrogen Energy, vol. 39, no. 8, pp. 4005-4008, 2014; doi:

10.1016/j.ijhydene.2013.06.078

[4] A. R. Møller, "Systems identification using pseudorandom noise applied to a sensorineural system," Computers \& Mathematics with Applications, vol. 12, no. 6, pp. 803-814, 1986; doi: 10.1016/08981221(86)90065-9

[5] P. Symons, Digital waveform generation. Cambridge, U.K.: Cambridge University Press, 2014.

[6] J. P. Schmidt, T. Chrobak, M. Ender, J. Illig, D. Klotz, and E. Ivers-Tiffée, "Studies on $\mathrm{LiFePO} 4$ as cathode material using impedance spectroscopy," Journal of Power Sources, vol. 196, no. 12, pp. 5342-5348, 2011; doi: 10.1016/j.jpowsour.2010.09.121

[7] M. Schönleber and E. Ivers-Tiffée, "Approximability of impedance spectra by RC elements and implications for impedance analysis," Electrochemistry Communications, vol. 58, pp. 15-19, 2015; doi:

10.1016/j.elecom.2015.05.018

[8] Raul Land, "Time-frequency impedance spectroscopy: excitation considerations," in IMEKO TC4 International Symposium on Novelties in Electrical Measurements and Instrumentations, vol. 1 of 15th IMEKO TC4 Internatinal Symposium on Novelties in Electrical Measurements and Instrumentations Proceedings, pp. 328-221.

[9] F. J. Harris, "On the use of windows for harmonic analysis with the discrete Fourier transform," Proc. IEEE, vol. 66, no. 1, pp. 51-83, 1978; doi:

10.1109/PROC.1978.10837

[10] N. Lohmann, P. Haussmann, P. Wesskamp, J. Melbert, and T. Musch, "Employing Real Automotive Driving Data for Electrochemical Impedance Spectroscopy on Lithium-Ion Cells," SAE Int. J. Alt. Power, vol. 4, no. 2, 2015; doi: 10.4271/2015-01-1187 